05,01

\title{
Ферромагнитный резонанс в монодоменной частице с одноосной анизотропией при продольной накачке
}

\author{
() Т.М. Василевская, Д.И. Семенцов , А.М. Шутый \\ Ульяновский государственный университет, \\ Ульяновск, Россия \\ ๑ E-mail: sementsovdi42@mail.ru
}

Поступила в Редакцию 28 сентября 2021 г.

В окончательной редакции 28 сентября 2021 г.

Принята к публикации 10 октября 2021 г.

На основе анализа прецессионной динамики намагниченности монодоменной магнитоодноосной частицы эллипсоидальной формы в спектре ферромагнитного резонанса, отвечающего случаю подмагничивания вдоль „трудной“ оси, при продольной накачке слабым высокочастотным полем выявлен дополнительный резонансный пик вблизи поля эффективной анизотропии $H_{\mathrm{KN}}$. Появление указанного пика связано с проявлением угловой бистабильности, возникающей из-за наличия двух симметричных угловых положений равновесия намагниченности частицы в поле, меньшем поля $H_{\mathrm{KN}}$.

Ключевые слова: ферромагнитный резонанс, „трудная“ плоскость, продольная накачка, бистабильность, эффективная анизотропия, прецессионная траектория, высокочастотный отклик.

DOI: 10.21883/FTT.2022.02.51948.214

\section{1. Введение}

Особенности динамического поведения дипольных решеточных структур связаны не только с дискретным, пространственным упорядочением наночастиц и характером их взаимодействия, но также с магнитными свойствами отдельных частиц, составляющих решетку. Прецессионная динамика магнитного момента отдельной монодоменной микро- и наночастицы во многом определяется ее размерами, симметрией, магнитной анизотропией, а также величиной и ориентацией внешних статического и переменного полей [1-8].

Одним из проявлений динамики магнитного момента (MM) в высокочастотном поле является ферромагнитный резонанс (ФМР) [9-11]. Известно, что для магнитоодноосной пленки, намагниченной в своей плоскости вдоль „трудной“ оси, в спектре ФМР на низких частотах имеются две резонансные ветви с разным характером зависимости частоты от подмагничивающего поля [10]. При значении поля, где обе ветви смыкаются, резонансная частота стремится к нулю. Для конечного значения частоты при указанном значении поля, согласно теории, резонансный пик отсутствует. Между тем, в работе [12] такой пик экспериментально обнаружен, а в работе [13] было показано, что появление этого резонансного пика связано с проявлением угловой бистабильности в прецессионном движении ММ пленки.

Поскольку характер основных резонансных зависимостей во многом является общим для образцов разного состава, геометрии, анизотропии, считаем, что подобного рода резонансные особенности могут проявляться в спектрах ФМР отдельной монодоменной одноосной микро- или наночастицы и, следовательно, в решетках из таких частиц. В настоящей работе на основе численного решения уравнения Ландау-Лифшица и анализа прецессионной динамики ММ однородно намагниченного эллипсоидального образца показано, что наличие двух близких угловых положений равновесия при подмагничивании в „трудном“ направлении полем, значение которого близко к полю эффективной анизотропии $H_{\mathrm{KN}}$, приводит к резонансному отклику и бистабильности прецессии ММ даже при продольной накачке слабым высокочастотным полем (т. е. при $\mathbf{h} \| \mathbf{H}_{0}$ и $\left.h \ll H_{0}\right)$. При этом величина резонансного отклика оказывается даже сильнее, чем при поперечной накачке (когда $\mathbf{h} \perp \mathbf{H}_{0}$ ). Отметим также, что при используемых в работе амплитуде и частоте поля накачки однородная мода сильно удалена по частоте от спин-волновой моды, поэтому отсутствует передача энергии от однородной прецессии спиновым волнам и нет развития спин-волновых неустойчивостей [14-16].

\section{2. Общие соотношения}

Рассмотрим образец, имеющий форму вытянутого эллипсоида вращения. Будем считать, что наряду с анизотропией формы у образца присутствует слабая наведенная одноосная анизотропия, легкая ось которой совпадает с осью симметрии образца. В этом случае плотность свободной энергии содержит зеемановскую энергию, энергии анизотропии и полей рассеяния

$$
F=-\mathbf{M}\left(\mathbf{H}_{0}+\mathbf{h}\right)+K \sin ^{2} \theta+\frac{1}{2} \mathbf{M} \widehat{N} \mathbf{M}
$$

Здесь: $K-$ константа одноосной анизотропии, $\theta-$ полярный угол вектора $\mathbf{M}$, отсчитываемый от оси 
симметрии эллипсоида (ось $O Z), \widehat{N}$ - диагональный тензор, компоненты которого связаны соотношением $N_{x}+N_{y}+N_{z}=4 \pi$ и зависят от отношения продольной и поперечной полуосей эллипсоида $n=l_{\|} / l_{\perp}[10]$. Для вытянутого эллипсоида вращения $n>1, N_{x}=N y=N_{\perp}$, $N_{z}=N_{\|}$и

$$
\begin{aligned}
\Delta N & =N_{\perp}-N_{\|} \\
& =2 \pi\left\{1-\frac{3}{n^{2}-1}\left[\frac{n}{\sqrt{n^{2}-1}} \ln \left(n+\sqrt{n^{2}-1}\right)-1\right]\right\} .
\end{aligned}
$$

Отдельные компоненты тензора $\widehat{N}$ могут быть записаны в виде

$$
N_{\|}=\frac{4 \pi}{3}-\frac{2}{3} \Delta N, \quad N_{\perp}=\frac{4 \pi}{3}+\frac{1}{3} \Delta N .
$$

Временна́я зависимость ориентации вектора и особенности его прецессионной динамики для различных случаев подмагничивания и высокочастотной накачки частицы определяются на основе численного решения уравнения Ландау-Лифшица, записанного в сферических координатах [11]:

$$
\begin{gathered}
\frac{\partial \theta}{\partial t}=-\frac{\gamma \alpha}{M} \frac{\partial F}{\partial \theta}-\frac{\gamma}{M \sin \theta} \frac{\partial F}{\partial \varphi}, \\
\frac{\partial \varphi}{\partial t}=\frac{\gamma}{M \sin \theta} \frac{\partial F}{\partial \theta}-\frac{\gamma \alpha}{M \sin ^{2} \theta} \frac{\partial F}{\partial \varphi},
\end{gathered}
$$

где $\alpha$ - безразмерная константа затухания, а плотность свободной энергии образца определена соотношением (1). В общем случае частота резонансной прецессии намагниченности определяется общим выражением

$$
\omega_{\text {res }}=\frac{\gamma}{M \sin \theta}\left[\left(\frac{\partial^{2} F}{\partial \varphi^{2}}\right)_{0}\left(\frac{\partial^{2} F}{\partial \theta^{2}}\right)_{0}-\left(\frac{\partial^{2} F}{\partial \varphi \partial \theta}\right)_{0}^{2}\right]^{1 / 2}
$$

где вторые производные от свободной энергии вычисляются для равновесных значений углов $\varphi_{0}$ и $\theta_{0}$, которые находятся из условия $\partial F / \partial \varphi=\partial F / \partial \theta=0$.

При подмагничивании вытянутого эллипсоида вдоль оси симметрии $\left(\mathbf{H}_{0} \| \mathbf{n}\right)$ равновесный угол $\theta_{0}$ равен нулю при любых значениях поля $H_{0}$, а резонансная зависимость в этом случае определяется высокочастотной ветвью ФМР:

$$
\begin{aligned}
\omega_{\mathrm{res}} & =\gamma\left(H_{0}+H_{\mathrm{KN}}\right) \\
& =\gamma\left[H_{0}+2 K / M_{0}+\left(3 N_{\perp}-4 \pi\right) M_{0}\right],
\end{aligned}
$$

где $\gamma=1.76 \cdot 10^{7}(\mathrm{Oe} \cdot \mathrm{s})^{-1}, H_{\mathrm{KN}}=2 K / M_{0}+\Delta N M_{0}-$ поле эффективной анизотропии.

При подмагничивании эллипсоида в „трудной“6 плоскости $\mathbf{H}_{0} \perp \mathbf{n}$ в равновесном состоянии вектор $\mathbf{M}_{0}$ лежит в плоскости векторов $\left(\mathbf{n}, \mathbf{H}_{0}\right)$. При этом отсчитываемый от направления поля $\mathbf{H}_{0}$ (например, от оси $O X$ ) равновесный азимутальный угол $\varphi_{0}=0$, а угол $\theta_{0}$ определяется выражениями

$$
\sin \theta_{0}= \begin{cases}1, & H_{0}>H_{\mathrm{KN}} \\ \pm \frac{H_{0}}{H_{\mathrm{KN}}}, & H_{0}<H_{\mathrm{KN}}\end{cases}
$$

Полевые зависимости резонансных частот в рассматриваемых случаях с учетом (1) и (5) определяются выражениями

$$
\omega_{\mathrm{res}}=\left\{\begin{array}{ll}
\gamma \sqrt{H_{\mathrm{KN}}^{2}-H_{0}^{2}}, & H_{0}<H_{\mathrm{KN}} \\
\gamma \sqrt{H_{0}\left(H_{0}-H_{\mathrm{KN}}\right)}, & H_{0}>H_{\mathrm{KN}}
\end{array} .\right.
$$

Из (8) следует, что одна из резонансных ветвей лежит в области полей $H_{0}<H_{\mathrm{KN}}$, другая - в области $H_{0}>H_{\mathrm{KN}}$, поэтому одному значению частоты $\omega<\omega_{0}$ отвечают два значения резонансного поля:

$$
H_{\mathrm{res}}^{(1,2)}=\left\{\begin{array}{l}
\sqrt{H_{\mathrm{KN}}^{2}-\omega^{2} / \gamma^{2}} \\
\frac{1}{2}\left(H_{\mathrm{KN}}+\sqrt{H_{\mathrm{KN}}^{2}+4 \omega^{2} / \gamma^{2}}\right)
\end{array} .\right.
$$

\section{3. Условия однородности намагниченности}

Вначале укажем на ограничения, накладываемые на размер частицы, динамику ММ которой мы рассматриваем. Здесь и далее использовались параметры материала частицы, близкие к характеристикам пермаллоя 80Ni20Fe: $4 \pi M_{0}=10^{4} \mathrm{Gs}, K=10^{4} \mathrm{erg} / \mathrm{cm}^{3}, \sigma \approx 10^{18} \mathrm{~s}^{-1}$, $\alpha=0.01$, частота СВЧ-поля $\omega=2 \cdot 10^{8} \mathrm{~s}^{-1}$ и $n=1.02$. Чтобы сохранялось однородное распределение намагниченности по образцу в присутствии СВЧ поля необходимо, чтобы максимальный размер частицы $d$ был намного меньше глубины скин-слоя $\delta=c /(2 \pi \sigma \omega \mu)^{1 / 2}$ [18]. Для указанных значений параметров и $c=3 \cdot 10^{10} \mathrm{~cm} / \mathrm{s}$, $\mu \approx 100$ получаем $d \ll \delta \approx 10^{-4} \mathrm{~cm}$.

Следующее ограничение накладывается из-за наличия тепловых флуктуаций, которые могут нарушать прецессионную динамику, определяемую в каждый момент времени действующим на ММ эффективным полем. Влияние тепловых флуктуаций описывается экспоненциальным множителем $\exp (\Delta U / k T)$ [19], где $\Delta U=\Delta N^{\mathrm{ef}} M_{0}^{2} V / 2$ - потенциальный барьер, разделяющий направления вдоль, ,легкой“ оси и в „трудной“ плоскости, $V$ - объем частицы, $\Delta N^{\mathrm{ef}}=H_{\mathrm{KN}} / M_{0}-$ параметр эффективной анизотропии. Чтобы тепловое возбуждение не нарушало прецессионную динамику, необходимо выполнение условия $V>V_{\min }$ [5], где $V_{\min }=2 k T / N^{\mathrm{ef}} M_{0}^{2} \approx 10^{-18} \mathrm{~cm}^{3}$. Таким образом, для частицы сферической (или близкой к сферической) ее диаметр $d>d_{\min } \approx 10 \mathrm{~nm}$. 
Еще одно ограничение связано с требованием однодоменности частицы, которое при дальнейшем рассмотрении считается выполненным. Для однодоменной частицы ее радиус должен быть меньше $R_{\mathrm{cr}} \approx \sigma_{s} / M_{0}^{2}$, где поверхностная энергия доменной границы для пермаллоя $\sigma_{s} \approx 1 \mathrm{erg} / \mathrm{cm}^{2}$. Поэтому для исследуемой нами частицы необходимо условие $d<2 R_{\mathrm{cr}} \approx 30 \mathrm{~nm}$. Согласно проведенным оценкам, оптимальным размером частицы для наблюдения ФМР является $10<d<30 \mathrm{~nm}$. Отметим однако, что по данным работы [20] металлические цилиндрические частицы с диаметром $d \approx 40-50 \mathrm{~nm}$ и высотой $h \approx 45 \mathrm{~nm}$ следует считать однодоменными.

\section{4. Численный анализ}

На рис. $1(a, b)$ приведены полевые зависимости равновесного угла $\theta_{0}$ и резонансных частот, построенные по
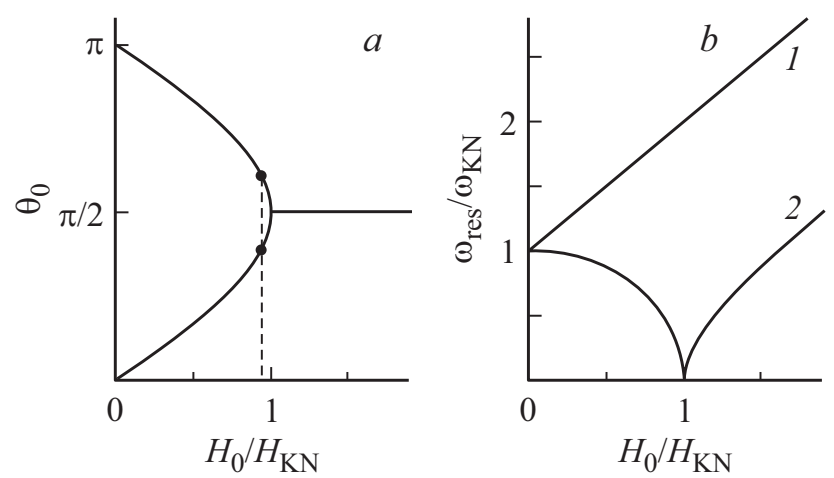

Рис. 1. Полевые зависимости $a$ ) равновесного угла $\theta_{0}$ и $b)$ резонансной частоты при подмагничивании вытянутого эллипсоида в „трудной“ плоскости (кривая 2), кривая 1 подмагничивание вдоль ,легкой“ оси. формулам (6-8). Вид зависимости $\theta_{0}\left(H_{0}\right)$ показывает, что в области $H_{0} \geq H_{\mathrm{KN}}$ имеется одна равновесная ориентация намагниченности $\left(\theta_{0}=\pi / 2, \mathbf{M} \| \mathbf{H}_{0}\right)$, тогда как в области $H_{0}<H_{\mathrm{KN}}$ намагниченность эллипсоида имеет два симметрично расположенных угловых положения равновесия. Если значение подмагничивающего поля оказывается близким к $H_{\mathrm{KN}}$, то угловое расстояние между двумя симметричными положениями равновесия также является малым и легко может преодолеваться под воздействием высокочастотного поля. При понижении частоты значения резонансных полей сближаются, и при $\omega \rightarrow 0$ обе ветви смыкаются в точке $H_{0}=H_{\mathrm{KN}}$.

Численное решений динамических уравнений проводилось с помощью метода Рунге-Кутта. Здесь и далее использовались параметры частицы, близкие к характеристикам пермаллоя состава $80 \mathrm{Ni} 20 \mathrm{Fe}\left(4 \pi M_{0}=10^{4} \mathrm{Gs}\right.$, $\left.K=10^{4} \mathrm{erg} / \mathrm{cm}^{3}, \alpha=0.01, n=1.02\right)$, частота СВЧ-поля $\omega=2 \cdot 10^{8} \mathrm{~s}^{-1}$, параметры статического поля выбирались близкими к резонансным значениям при заданных условиях подмагничивания и накачки. Временная зависимость поля накачки задается в виде $h(t)=h \sin \omega t$, где $h=0.2 \mathrm{Oe}$, что при условии $h \ll H_{0}$ предполагает линейный характер ФМР. При подмагничивании вытянутого эллипсоида вдоль оси симметрии $\left(\mathbf{H}_{0} \| \mathbf{n}\right)$ и накачке полем $\mathbf{h} \perp \mathbf{H}_{0}$, прецессионная траектория вектора $\mathbf{M}$ выходит из начального положения $\varphi_{0}=\theta_{0}=0$ на круговую траекторию, которая на плоскости $\left(\mu_{x}, \mu_{y}\right)$, где $\mu_{\alpha}=M_{\alpha} / M_{0}$, представлена на рис. 2 .

При подмагничивании в „трудной“ плоскости $\left(\mathbf{H}_{0} \perp \mathbf{n}\right)$ прецессионная траектория становится более сложной, причем на ее форму могут существенно влиять как величина статического поля и частота, так и амплитуда и ориентация переменного поля. Традиционной реализации ФМР отвечает ортогональность статического $\mathbf{H}_{0}$ и высокочастотного h полей [4]. Ниже
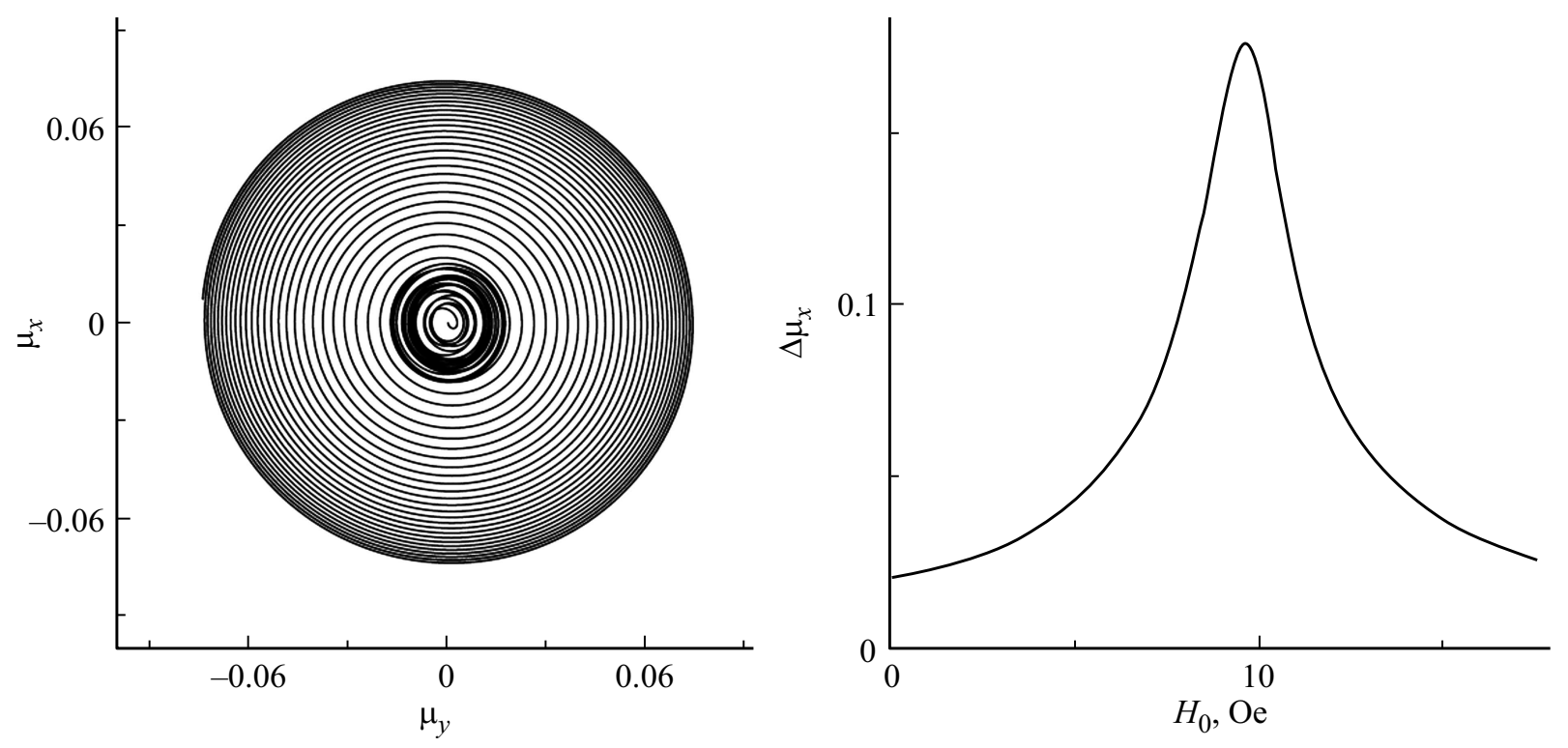

Рис. 2. Проекция прецессионной резонансной траектории при подмагничивании вдоль „легкой“ оси эллипсоида $\left(\mathbf{h} \perp \mathbf{H}_{0} \| \mathbf{n}\right)$. 

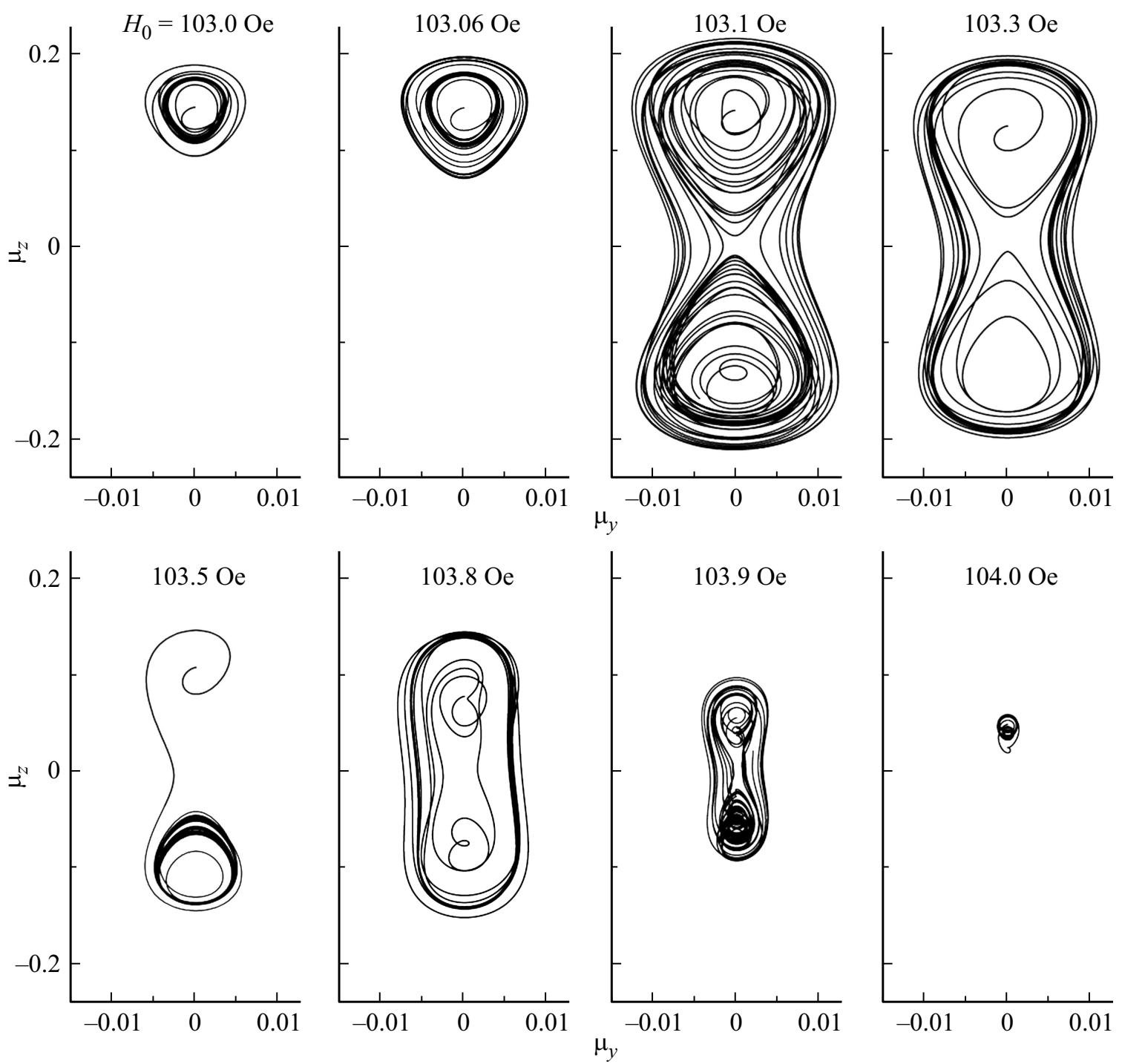

Рис. 3. Проекции на плоскость $Z O Y$ прецессионных траекторий при различных полях подмагничивания в „трудной“ плоскости эллипсоида и продольной накачке $\left(\mathbf{h} \| \mathbf{H}_{0}\right)$.

мы рассмотрим прецессию намагниченности эллипсоида при продольной накачке $\left(\mathbf{h}\left\|\mathbf{H}_{0}\right\| O X\right)$ и слабом высокочастотном поле $\left(h \ll H_{0}\right)$. В этом случае свободная энергия имеет вид

$$
\begin{aligned}
F= & -M\left(H_{0}+h \sin \omega t\right) \cos \varphi \sin \theta+K \sin ^{2} \theta \\
& +\frac{M^{2}}{6}\left[4 \pi+\left(3 \sin ^{2} \theta-2\right) \Delta N\right] .
\end{aligned}
$$

На рис. 3 приведены проекции на плоскость ZOY; прецессионных траекторий вектора $\boldsymbol{\mu}$, построенные для нескольких значений $H_{0}$, близких к резонансным, с момента включения поля до установления практически стационарного движения. На выбранной частоте в соответствии с (9) резонансные значения поля $\mathbf{H}_{\text {res }}=(103.45,105.35)$ Ое. Первое значение отвечает случаю $H_{0}<H_{\mathrm{KN}}$, когда направление равновесного век- тора $\mathbf{M}_{0}$ не совпадает с $\mathbf{H}_{0}$. При $H_{0}=H_{\mathrm{KN}}=104.116$ Oе вектор $\mathbf{M}_{0}$ становится параллельным полю $\mathbf{H}_{0}$.

Из представленных на рисунке траекторий видно, что с ростом поля $H_{0}$ вначале наблюдается резкое возрастание амплитуды прецессии и достижение максимума отклика при $H_{0}=103.1$ Ое. Далее, на малом интервале поля $\Delta H_{0} \approx 1 \mathrm{Oе}$, наряду с изменением характера, происходит уменьшение амплитуды траекторий, а при значении поля $H_{0} \simeq 104 \mathrm{Oe}-$ резкий спад амплитуды практически до нулевого значения. Отметим, что в узкой области поля вблизи значения $H_{0} \simeq 103.5 \mathrm{Oe}$ проявляется нестабильность, которая состоит в изменении характера траектории: прецессия стартует из одного положения равновесия, а установившаяся траектория оказывается „притянута“ симметричным (относительно „трудной“ плоскости) равновесным положением. При этом амплитуда такой прецессии существенно меньше амплитуды прецессии возле двух положений равновесия. 

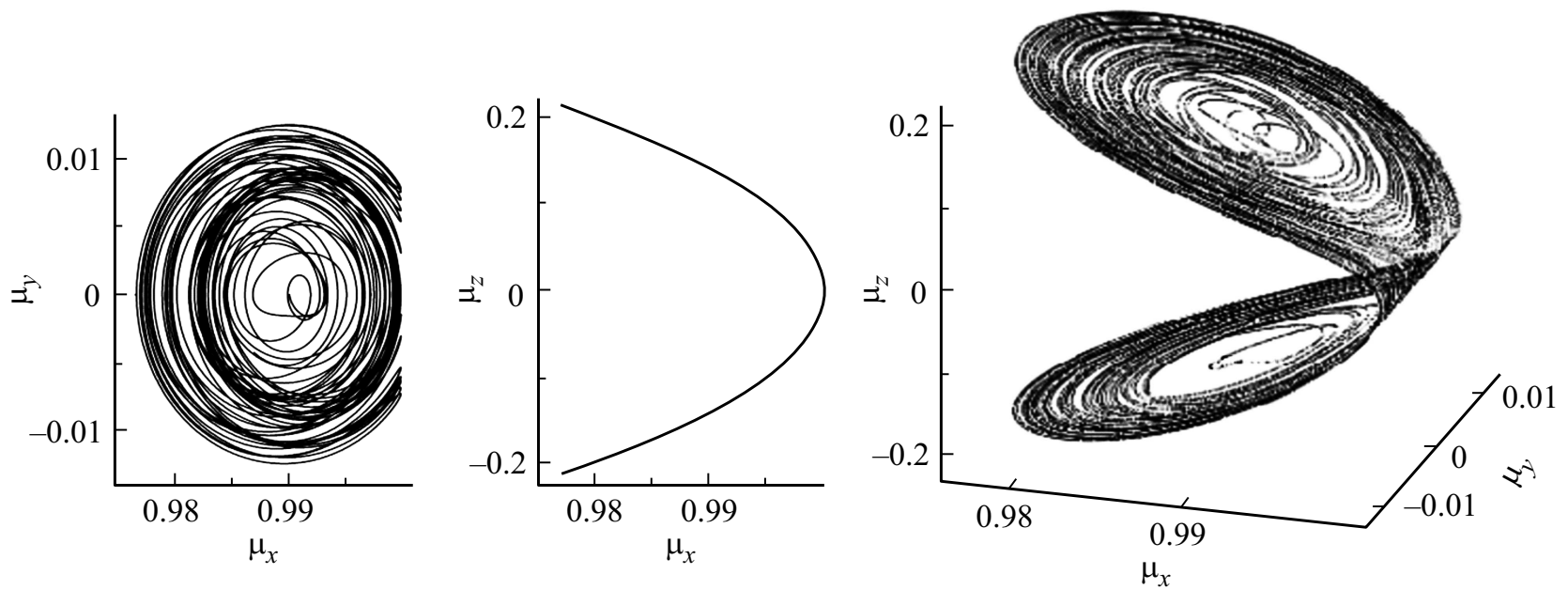

Pис. 4. Две проекции и общий вид резонансной траектории при подмагничивании в „трудной“ плоскости и продольной накачке $\left(\mathbf{h} \| \mathbf{H}_{0} \perp \mathbf{n}\right)$.

При $H_{0} \geq H_{\mathrm{KN}}$ прецессия под действием продольного поля накачки исчезает. Таким образом, высокоамплитудная прецессия намагниченности вблизи резонансного значения статического поля при слабой продольной накачке связана с малыми отклонениями равновесной намагниченности от направления статического поля и наличием малой компоненты намагниченности, ортогональной СВЧ полю при $H_{0}<H_{\mathrm{KN}}$.

На рис. 4 для поля $H_{0}=103.1$ Ое приведены еще две проекции вектора $\boldsymbol{\mu}$ и пространственный вид траектории прецессии с максимальной амплитудой при подмагничивании в „трудной“ плоскости и продольной накачке (еще одна проекция этой траектории приведена на рис. 3). В отличие от плоской траектории при подмагничивании вдоль „легкой“ оси (рис. 2), в рассматриваемом случае установившаяся траектория является более сложной объемной замкнутой кривой.

При этом изменение продольной компоненты $\mu_{z}$ для рассматриваемой траектории более чем на порядок выше изменения двух поперечных компонент.

Известно, что отклик магнитной системы на воздействие переменного поля определяется мнимой частью высокочастотной восприимчивости $\chi^{\prime \prime}$, которая связана с поглощаемой мощностью СВЧ-поля соотношением $P=\chi^{\prime \prime} \omega h^{2} V / 2$, где $V$ - объем образца. Так как частота, амплитуда поля, а также объем образца считаем заданными, то высокочастотный отклик, определяемый величиной $\chi^{\prime \prime}$, с достаточной степенью точности можно связать с величиной $\Delta \mu_{z}=\left|\mu_{z}^{\max }-\mu_{z}^{\min }\right|$.

С учетом сказанного, на рис. 5 приведена полевая зависимость величины вытянутого эллипсоида при его подмагничивании в „трудной“ плоскости для двух случаев накачки - продольной и поперечной (сплошная и штриховая линии). Указанные зависимости отличаются числом „резонансных“ пиков: при традиционной (для линейного ФМР) поперечной накачке таких пиков два, тогда как при продольной накачке пик один. При при-

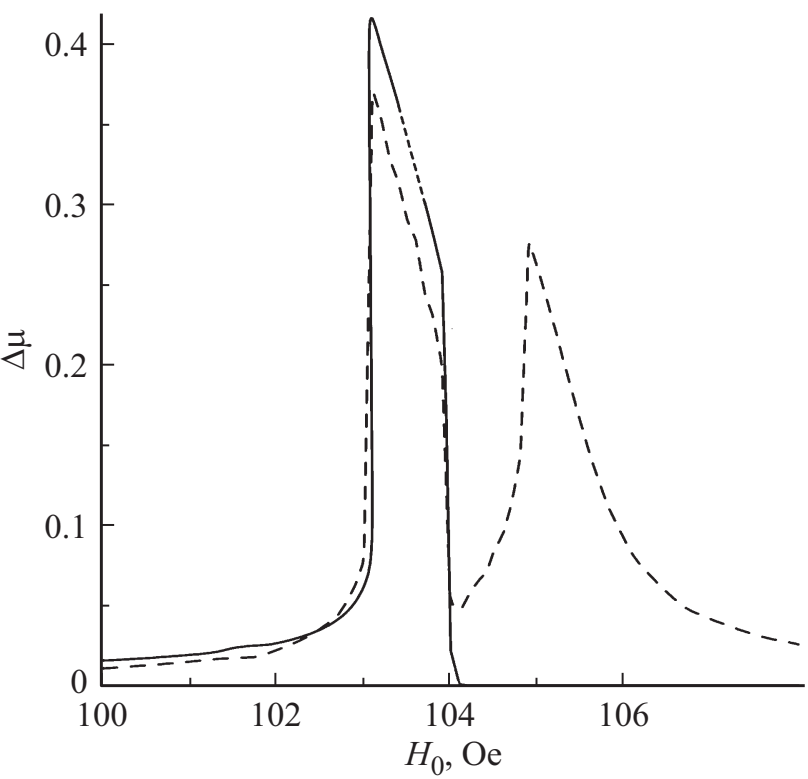

Рис. 5. Спектр ФМР вытянутого эллипсоида при подмагничивании в „трудной“ плоскости, продольной и поперечной накачке (сплошная и штриховая линии).

ближении к полю бистабильности $H_{b}$, величина которого определяется параметрами частицы и поля накачки (в рассматриваемом случае $H_{b}=103.1 \mathrm{Oe}$ ), происходит резкий рост величины $\Delta \mu_{z}$ с достижением максимума отклика. Как уже отмечалось, этот максимум обусловлен бистабильностью, которая связана с двумя близкими по углу равновесными состояниями в области $H_{0}<H_{\mathrm{KN}}$. Эти состояния при любой ориентации поля $\mathbf{h}(t)$,притягивают“ магнитный момент, и его прецессия с разной вероятностью (в зависимости от близости статического поля к значению $H_{b}$ ) происходит около каждого из них. Затем с увеличением поля происходит асимметричный 
спад отклика, который при $H_{0} \simeq 104 \mathrm{Oе}$ резко падает практически до нуля, что означает отсутствие прецессии.

Аналогичное поведение отклика в области $H_{0}<H_{\mathrm{KN}}$ наблюдается и при поперечной накачке (штриховая линия). Однако в этом случае амплитуда отклика меньше, чем в случае продольной накачки, а также, кроме указанного пика, в спектре присутствует пик в области $H_{0}>H_{\mathrm{KN}}$. Его амплитуда меньше амплитуды первого пика. Отметим также, что амплитуда отклика при подмагничивании в „трудной“ плоскости существенно выше амплитуды отклика при подмагничивании вдоль оси симметрии эллипсоида. При этом ширина резонансных пиков $\Delta H_{0} \simeq 1 \mathrm{Oе}$, что значительно у́же ширины резонансной линии при подмагничивании вдоль ,легкой“ оси (см. рис. 2, $b$ ).

\section{5. Заключение}

Проведенный анализ показывает, что в спектре ФМР монодоменной магнитоодноосной микро- или наночастицы с формой вытянутого эллипсоида вращения при подмагничивании в „трудной“ плоскости и продольной накачке слабым СВЧ-полем $\left(\mathbf{h} \| \mathbf{H}_{0} \perp \mathbf{n}\right)$ в области $H_{0}<H_{\mathrm{KN}}$ наблюдается резонансный пик, положение которого не отвечает резонансному значению поля $H_{\text {res }}^{(1)}$, амплитуда выше амплитуды резонансного пика при поперечной накачке, а прецессионная траектория намагниченности имеет сложный объемный характер с двумя „центрами притяжения“. Ширина этого пика значительно у́же ширины резонансной кривой при поперечной накачке. Появление пика и бистабильности связано с наличием двух близких по углу равновесных состояний магнитного момента эллипсоида. Подобного рода пики должны проявляться также в спектрах ФМР сплюснутых эллипсоидальных частиц с одноосной анизотропией и в структурах с кубической кристаллографической анизотропией. О наблюдении подобных пиков в резонансных исследованиях тонких магнитных пленок и их интерпретации сообщалось в работах $[13,14]$.

\section{Конфликт интересов}

Авторы заявляют, что у них нет конфликта интересов.

\section{Список литературы}

[1] E.Z. Meilikhov, R.M. Farzetdinova. JMMM 268, 1-2, 237 (2004).

[2] Р.Б. Моргунов, А.И. Дмитриев, Г.И. Джардималиева, А.Д. Помогайло, А.С. Розенберг, Y. Tanimoto, M. Leonowicz, E. Sowka. ФTT 49, 8, 1436 (2007).

[3] A. Sukhov, J. Berakdar. Phys. Rev. B 79, 13, 134433 (2009).

[4] Ю.И. Джежеря, К.О. Демишев, В.Н. Коренивский. ЖЭТФ 142, 2(8), 318 (2012).

[5] Е.3. Мейлихов, Р.М. Фарзетдинова. ФТТ 56, 12, 2329 (2014).
[6] А.М. Шутый, Д.И. Семенцов. Письма в ЖЭТФ 99, 12, 806 (2014).

[7] A.M. Shuty̌̌, S.V. Eliseeva, D.I. Sementsov. Phys. Rev. B 91, 2, 024421 (2015).

[8] T. Kiseleva, S. Zholudev, A. Novakova, T. Grigoryeva. Compos. Struct. 138, 12 (2016).

[9] I.S. Poperechny, Yu.L. Raikher. Phys. Rev. B 93, 1, 014441 (2016).

[10] Ферромагнитный резонанс. Сб. статей / Под ред. С.В. Вонсовского. Физматлит, М. (1961).

[11] А.Г. Гуревич, Г.А. Мелков. Магнитные колебания и волны. Наука, М. (1994).

[12] В.Г. Шавров, В.И. Щеглов. Динамика намагниченности в условиях изменения её ориентации. Физматлит, М. (2019).

[13] Б.А. Беляев, А.В. Изотов, С.Я. Кипарисов. Письма в ЖЭТФ 74, 4, 248 (2002).

[14] Т.М. Василевская, Д.И. Семенцов. ЖЭТФ 137, 5, 861 (2010).

[15] М.А. Шамсутдинов, Л.А. Калякин, А.Т. Харисов. ЖТФ 80, 6, $106(2010)$

[16] П.Е. Зильберман, А.Г. Темирязев, М.П. Тихомирова. ЖЭТФ 108, 1(7), 281 (1995).

[17] В.В. Тихонов, А.В. Толкачев. ФТТ 36, 1, 185 (1994).

[18] Л.Д. Ландау, Е.М. Лифшиц. Теоретическая физика. Наука, M. (1982). T. VIII.

[19] L. Neel. Ann. Geophys. (C.N.R.S.) 5, 99 (1949).

[20] С.А. Непийко. Физические свойства малых металлических частиц. Наук. думка, Киев (1985).

Редактор Е.В. Толстякова 